\title{
Early Weight Gain during Pregnancy: Which Women are the Most Affected?
}

Tebbani Fouzia, Oulamara Hayet, Agli Abdenacer

Institute of Nutrition, Food and Food Technologies (INATAA), Laboratory of Nutrition and Food Technologies (LNTA), Algeria

\begin{tabular}{l} 
Article Info \\
\hline Article history: \\
Received Feb 29, 2016 \\
Revised Mar 27, 2016 \\
Accepted Apr 29, 2016 \\
\hline Keyword: \\
First and second trimester \\
Pregnancy \\
pre-pregnancy BMI \\
Weight gain \\
Health and social status
\end{tabular}

Health and social status

\begin{abstract}
Maternal weight gain during pregnancy is a good prediction tool in short and long term health of pregnant women and their children. To study the effect of early weight gain of pregnant women until the end of the $2^{\text {nd }}$ trimester of pregnancy, depending on their pre-pregnancy body mass index. 116 healthy pregnant women were followed until the $2^{\text {nd }}$ trimester of pregnancy, their weight and height before pregnancy, as well as the current weight at the end of the $1^{\text {st }}$ and $2^{\text {nd }}$ trimesters were collected. Data included age, parity, eating habits and physical activity level. Statistics were performed using the Statview software. The mean pre-pregnancy BMI was $27 \pm 5.27 \mathrm{~kg} / \mathrm{m}^{2}$. Weight gain in the $2^{\text {nd }}$ trimester is $6.33 \pm 4.84 \mathrm{~kg}$. It decreases with the increasing age of the mother (25\% of women between 20 and 24.9 years vs $12.5 \%$ of more than 35$)$. Also, it decreases with the increasing number of children (62.5\% in nulliparous vs $25 \%$ in multiparous). Breakfast is skipped by $64.5 \%$ of overweight pregnant women in the 1 st trimester and $90 \%$ in the $2^{\text {nd }}$ one. $80.17 \%$ and $69.83 \%$ of pregnant women do not practice any physical activity. Overweight and obese pregnant women before pregnancy do not take enough weight during pregnancy. Prospects will to analyze behaviors related to health and social status.
\end{abstract}

Copyright (C) 2016 Institute of Advanced Engineering and Science. All rights reserved.

\section{Corresponding Author:}

Tebbani Fouzia,

Institute of Nutrition, Food and Food Technologies (INATAA),

Laboratory of Nutrition and Food Technologies (LNTA), Algeria.

E-mail: fouziatebani@yafoo.fr

\section{INTRODUCTION}

Rates of obesity have increased significantly over the past several decades. Today over $35 \%$ of reproductive age women are obese (Body Mass Index (BMI) $\geq 30 \mathrm{~kg} / \mathrm{m}^{2}$ ), and $8 \%$ are extremely obese (BMI $\geq 40 \mathrm{~kg} / \mathrm{m}^{2}$ ) [1]. The obesity epidemic is impacting pregnancy. Over half of all women entering pregnancy in the United States are either overweight or obese [2].

Numerous studies have shown obesity and excessive gestational weight gain (GWG) are associated with both maternal and fetal complications including gestational diabetes, hypertension, preeclampsia, fetal macrosomia, cesarean delivery, and postpartum weight retention [3],[4].

Weight gain during pregnancy is attributable to the uterus and its contents (foetus, amniotic fluid and placenta), breasts, blood and interstitial fluid. A smaller fraction of that weight gain is due to an increase in cellular water and deposition of new fat and protein constituting maternal reserves [5]. Though the range of weight gain considered normal is wide, it depends on the pre-gestational body mass index (BMI) [6]. The most widely accepted recommendations regarding GWG are those published by the Institute of Medicine (IOM) of the United States of America [7]. 
These guidelines state that healthy women who have a normal weight for their height (BMI 18.524.9) should gain 11.5-16 kg during pregnancy. Overweight women (BMI 25-29.9) should gain 7-11.5 kg and obese women (BMI greater than 30) should only put on 5-9 kg. It is also recommended to gain between 0.5 and $2 \mathrm{~kg}$ during the first trimester [4]. In the second trimester, underweight women should gain $0.5 \mathrm{~kg}$ each week of pregnancy, normal women $0.4 \mathrm{~kg} /$ week, overweight women $0.3 \mathrm{~kg} /$ week and obese women $0.2 \mathrm{~kg} /$ week [4].

Despite these guidelines, over $55 \%$ of obese women gain more than the recommended amount [2]. GWG is not linear over the course of pregnancy. Weight gain starts slowly in the first trimester and increases more consistently in the second and third trimesters [8]. The ability to identify women at risk for excessive gestational weight gain allows for weight management interventions.

The second half of pregnancy is characterized by progressive insulin resistance [9],[10], and maternal fat accumulation appears to influence subsequent maternal insulin resistance [9],[11]. The pattern of GWG is higher in the second trimester at a rate of $0.563 \mathrm{~kg}$ per week and birth weight is strongly correlated with weigh gain during that period [12].

Previous studies have suggested that excessive early GWG might be associated with an early increase in insulin resistance leading to exhaustion of the pancreatic B cell. This B cell depletion could reduce the capacity to compensate for the increasing insulin resistance of pregnancy, and therefore lead to hyperinsulinemia, maternal hyperglycemia and excessive fetal growth [9], [13]-[15].

However it is important to remember the proposed objectives by the Swedish study of 2007 which allow decreasing the number of caesarean sections but also of persistent overweight and obesity in 1 year of childbirth, without increasing the number of other complications. These objectives are a weight gain of $10 \mathrm{~kg}$ maximum, regardless of the initial Body Mass Index of the patient. A weight gain more than $10 \mathrm{~kg}$ at 6 months of pregnancy is deemed excessive (risk factor of gestational diabetes). It is to be modulated according to the pre-pregnancy BMI [16]. Our study assessed the effect of early weight gain of pregnant women until the end of the $2^{\text {nd }}$ trimester of pregnancy, depending on their pre-pregnancy body mass index.

\section{RESEARCH METHOD}

We conducted a prospective and a longitudinal cohort study nearby 116 pregnant women during the first and second trimesters of pregnancy in maternities, antenatal centers and private gynecologists in Constantine, Algeria from December 2013 to August 2015. The participants were recruited and followed-up longitudinally, as described previously. The trimesters were defined as first (less than 16 complete weeks), second (15-28 complete weeks). Women were recruited before 16 weeks of amenorrhea. Women were eligible for participation if they entered prenatal care before the 16s week of amenorrhea, aged 18 years old and more and were healthy and mentally competent. We excluded women refusing to participate in the study, missing information on pre-pregnancy weight, known diabetes, hypertension and anemia before pregnancy. Potentially eligible women were sent an informational letter explaining the study and its objectives and requesting their participation. Written consent was obtained from the mother at inclusion. At enrollment, a standardized questionnaire was completed. Data collected include maternal characteristics (age, parity), maternal pre-pregnancy weight, height, pre-pregnancy BMI, weight gain at the end of the first and second trimesters of pregnancy, eating habits (such as eating between meals, taking or not of breakfast and consuming more fried foods) and the practice of physical activity (at least walking). These informations were obtained by face to face interview with each pregnant woman. Weight and height were measured according to a standard protocol. Weights of pregnant women were self reported or recorded at the early first trimester during their first visit and continued in every trimester by using an electronic weighing balance Seca to the nearest $0.1 \mathrm{~kg}$. Height was measured in $\mathrm{cm}$ using a locally made anthropometer. The pregnant women were asked to maintain an upright and erect posture with her feet together and the back of her heels touching the pole of the anthropometer. The height was measured when the horizontal headpiece was lowered onto the women's head. We calculated pre-pregnancy BMI using either a documented first trimester weight and height or a self reported weight. Pre-pregnancy body mass index (BMI) was computed as reported weight $(\mathrm{kg})$ divided by square of measured height $(\mathrm{m})$ and categorized into four groups as underweight $(<18.5$ $\left.\mathrm{kg} / \mathrm{m}^{2}\right)$, normal $\left(\geq 18.5\right.$ and $\left.<25 \mathrm{~kg} / \mathrm{m}^{2}\right)$, overweight $\left(\geq 25\right.$ and $\left.<30 \mathrm{~kg} / \mathrm{m}^{2}\right)$ and obese $\left(\geq 30 \mathrm{~kg} / \mathrm{m}^{2}\right)$. Prepregnancy BMI and gestational weight gain were categorized based on Institute of Medicine BMI groups and gestational weight gain guidelines [4]. Weight (in $\mathrm{kg}$ ) at each prenatal visit (at the end of the first and the second trimesters) were collected. The weight gain variate was categorical and had 3 values: weight gain above, below, or within IOM recommendations. At the end of the first trimester of pregnancy, all women should gain between 0.5 and $2 \mathrm{~kg}$ [4]. In the second trimester, underweight women should gain $0.5 \mathrm{~kg}$ each week of pregnancy, normal women $0.4 \mathrm{~kg} / \mathrm{week}$, overweight women $0.3 \mathrm{~kg} /$ week and obese women $0.2 \mathrm{~kg} /$ week [4]. 
Statistical analysis was performed using Stat View software. Quantitative variables were analyzed by student's t-test and one way- ANOVA. Binary logistic regression analysis was done to find out the association among body mass index, gestational weight gain, some maternal factors such as age, height and parity. $\mathrm{P}$ values $<0.05$ were considered significant. The $\chi^{2}$ test was used in comparison analyses, whereas correlations were evaluated by Pearson and Spearman tests.

\section{RESULTS AND ANALYSIS}

The database contained 245 women, and, of these, 116 women met all inclusion criteria and were included in the study. We excluded women with missing or implausible information on pre-pregnancy BMI, pre-pregnancy weight, known diabetes, hypertension and anemia before pregnancy.

We identified 116 patients who received care through maternities, antenatal centers and private gynecologists in Constantine, Algeria from December 2013 to August 2015. All women initiated care at less than 16 weeks of amenorrhea and had a documented first trimester weight.

The socio-demographic and other characteristics (age, parity, pre-pregnancy body weight, height, body mass index and gestational age) of the 116 women in the analysis cohort are shown in Table 02 . Mean weight before pregnancy was $71 \pm 13.99 \mathrm{~kg}$, the overall mean pre-pregnancy BMI was $27.29 \pm 5.27 \mathrm{~kg} / \mathrm{m}^{2}$. GWG in the first trimester was $1.25 \pm 3.8 \mathrm{~kg}$ and in second trimester was $6.34 \pm 4.84 \mathrm{~kg}(0.53 \mathrm{~kg} / \mathrm{week})$. A majority of the women $43(37.07 \%)$ started pregnancy with a normal body mass index (BMI). The second largest BMI group was overweight $36(31.03 \%)$ and obese $35(30.17 \%)$. The majority of women in the sample were between 20 and 34 years of age $(81.03 \%)$.

Table 2. Distribution of participants according to clinical characteristics and weight gain $(\mathrm{n}=116)$

\begin{tabular}{llcc}
\hline \multicolumn{1}{c}{ Category } & \multicolumn{1}{c}{ M \pm SD } & Minimum & Maximum \\
\hline Age (years) & $30 \pm 5.00$ & 20 & 42 \\
$20-34.9$ & $81.03 \%$ & & \\
$\geq 35$ & $18.96 \%$ & 0 & 4 \\
Parity & $1 \pm 0.99$ & & \\
$\quad$ Nulliparity & $47(40.52 \%)$ & & \\
$\quad$ Primiparity & $36(31.03 \%)$ & 23 & 28 \\
$\quad$ Multiparity & $33(28.48 \%)$ & 45 & 114 \\
Pregnancy term & $27 \pm 1.13$ weeks of amenorrhea & 1.46 & 1.72 \\
Initial weight $(\mathrm{kg})$ & $71 \pm 13.99$ & 16.52 & 46.84 \\
Height $(\mathrm{m})$ & $1.61 \pm 0.06$ & -15 & 9 \\
Pre-pregnancy BMI $\left(\mathrm{kg} / \mathrm{m}^{2}\right)$ & $27.29 \pm 5.27$ & $-4,1$ & 17.2 \\
Weight gain $\left(1^{\mathrm{st}}\right.$ trimester) & $1.25 \pm 3.8$ & & \\
Weight gain $\left(2^{\text {nd }}\right.$ trimester) & $6.34 \pm 4.84$ & & \\
\hline
\end{tabular}

Data are mean \pm SD or $\mathrm{n}(\%)$ unless otherwise specified

In general, as pre-pregnancy BMI increased, the amount of weight gained during pregnancy decreased (7.96 $\pm 4.86 \mathrm{~kg}$ in normal weight women vs $4.58 \pm 4.14 \mathrm{~kg}$ in obese ones, $\mathrm{p}=0.0017)$ (Table 03).

The rate of maternal weight gain from the first to the second trimester of pregnancy was a significant predictor of excessive weight gain.

Table 3. Distribution of participants according to weight gain in the $1^{\text {st }}$ and $2^{\text {nd }}$ trimesters of pregnancy by pre-pregnancy state weight

\begin{tabular}{|c|c|c|}
\hline \multirow{2}{*}{ Pre-pregnancy state weight } & \multicolumn{2}{|c|}{ Weight gain $(\mathrm{M} \pm \mathrm{SD})$} \\
\hline & $1^{\text {st }}$ Trimester & $2^{\text {nd }}$ Trimester \\
\hline Underweight & $2.59 \pm 0.18$ & $7.55 \pm 0.78$ \\
\hline Normal & $1.66 \pm 3.22$ & $7.96 \pm 4.86$ \\
\hline Overweight & $1.1 \pm 3.47$ & $6.04 \pm 5.06(\mathrm{P}=0.09)$ \\
\hline Obese & $0.83 \pm 4.81$ & $4.58 \quad 4.14(\mathrm{P}=0.0017)$ \\
\hline
\end{tabular}

Second- GWG rate varied with many sociodemographic characteristics, including maternal age and parity. In contrast, first-trimester GWG rate did not vary with these characteristics, but was associated with behaviors including, pregnancy physical activity, and diet pattern. GWG rate varied across pre-pregnancy BMI categories for second trimester gain but not for first-trimester gain.

Among the 116 women analyzed, $62.5 \%$ of women with a normal pre-pregnancy BMI, $25 \%$ of overweight and $12,5 \%$ of obese women had an excessive early gestational weight gain $(\mathrm{p}=0.02)$ (Figure 1). 


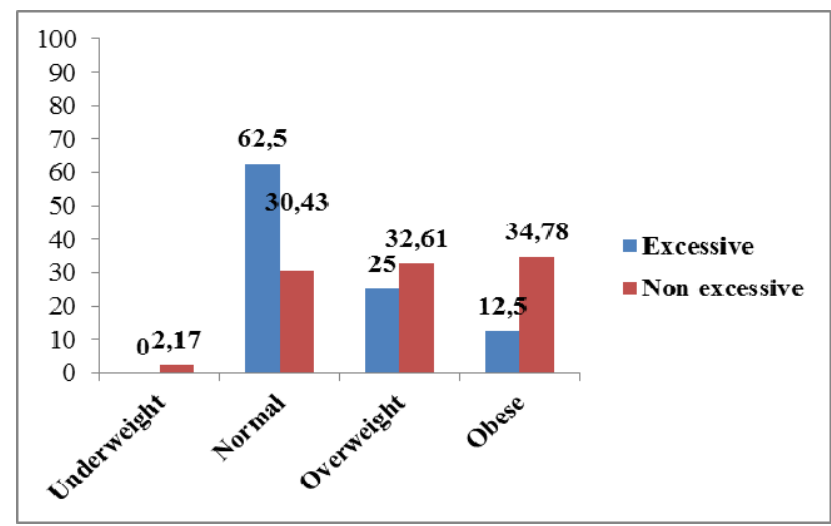

Figure 1. Pre-pregnancy state weight and $2^{\text {nd }}$ trimester weight gain

Gestational weight gain decreased with the increasing age of the mother $(25 \%$ of women between 20 and 24.9 years vs $12.5 \%$ in women of more than 35 years old, $\mathrm{p}=0.07$ ) (Figure 3 ).

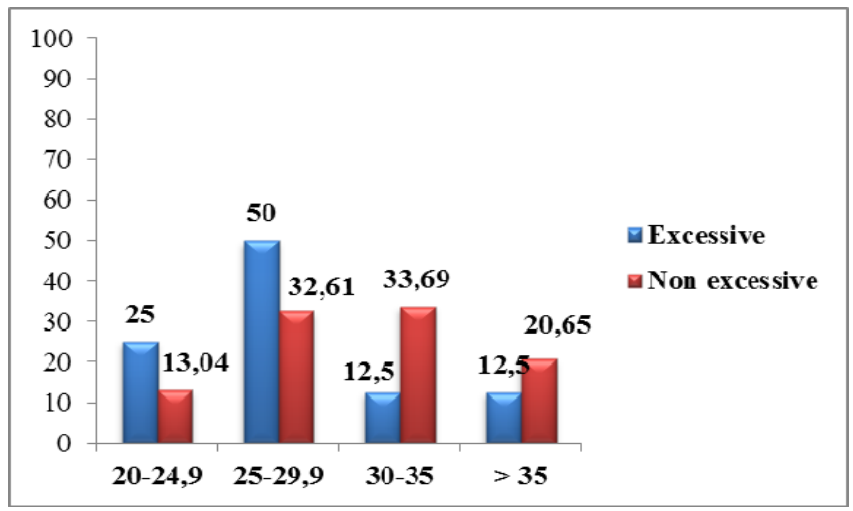

Figure 2. Distribution of pregnant women weight gain ( $2^{\text {nd }}$ trimester) according to age

As shown in Figure 3, $62.5 \%$ of nulliparous, $12.5 \%$ of primiparous and $25 \%$ of multiparous women had excessive weight gain in the 2 nd trimester. Weight gain decreases with the increasing number of children ( $62.5 \%$ in nulliparous women vs $25 \%$ in multiparous, $\mathrm{p}=0.02)$.

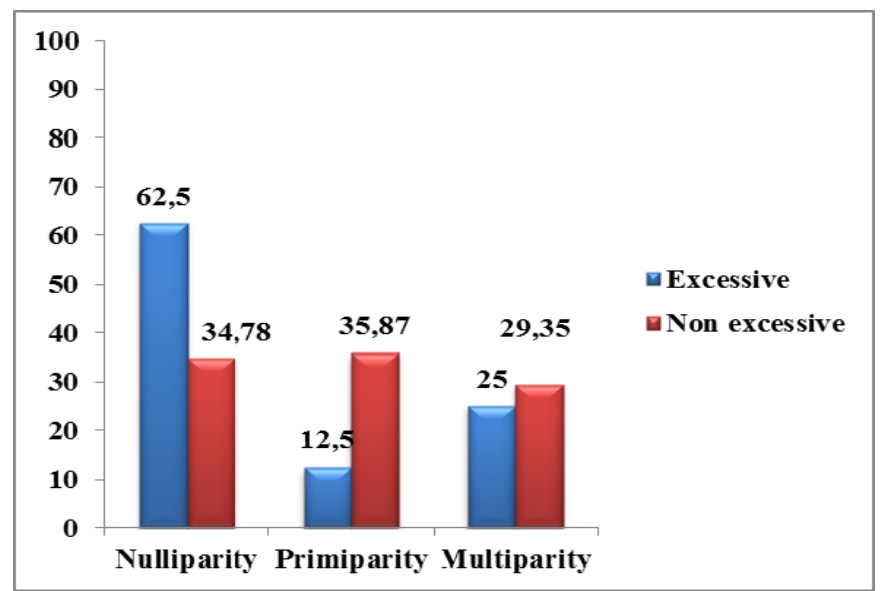

Figure 3. Distribution of pregnant women weight gain ( $2^{\text {nd }}$ trimester) according to parity 
GWG was significantly lower in older, more parous women, with age and parity strongly related $(\mathrm{p}=0.01, \mathrm{p}=0.07$ ) (Figure 2 and 3). Maternal weight gain during pregnancy decreased with increasing prepregnancy BMI, and obese women differed from their peers with a mean of $4 \mathrm{~kg}$ gained vs. about $8 \mathrm{~kg}$ for the other women. Also, obese women were also less likely to be nulliparous than women with a normal BMI.

Breakfast is skipped by $64.5 \%$ of overweight pregnant women in the 1 st trimester and $90 \%$ in the $2^{\text {nd }}$ one. A percentage of $80.17 \%$ (of which $63.41 \%$ are overweight) and $69.83 \%$ (of which $59.26 \%$ are overweight) of pregnant women do not practice any physical activity.

\subsection{Discussion}

In this study, $62.5 \%$ of women with a normal pre-pregnancy BMI, $25 \%$ of overweight and $12.5 \%$ of obese women had an excessive early gestational weight gain $(p=0.02)$ greater than the 2009 IOM recommendations.

In 2009, the U.S. Institute of Medicine (IOM) published revised guidelines for gestational weight gain. The goal of these guidelines is to optimize both maternal and child outcomes, and to decrease the rates of both maternal postpartum and childhood obesity [17]. These guidelines do not specifically address the timing of excessive weight gain during the pregnancy (early vs. late).

As obesity becomes more prevalent in pregnancy, it is increasingly important for clinicians to monitor gestational weight gain. Our data suggest a divergence in weight gain patterns as early as $12-14$ weeks. Women who exceeded pregnancy weight recommendations started to accelerate their weight gain at the beginning of the second trimester and gained a majority of weight during that trimester. Women who gained more than two kgs at 12-14 weeks were likely to exceed gestational weight gain (96\% positive predictive value [95 \% CI 79-99\%]).

All obese women had minimal weight gain to no weight gain until mid-pregnancy. [18]. Another large study of over 58.000 women within a Danish National Birth Cohort showed a mean gestational weight gain for obese primiparous women of $11.3 \mathrm{~kg}$ and $9.9 \mathrm{~kg}$ for multiparous women. In this cohort $45 \%$ of obese women gained less than $10 \mathrm{~kg}$ and the mean weight gain decreased with increasing BMI [19].

Our results showed that weight gain in pregnancy was lower when pre-pregnancy BMI was elevated. In particular, women who were overweight or obese before pregnancy generally gained less weight during pregnancy and very few women had an excessive weight gain. However, during pregnancy, fat is stored to secure energy supply during fetal growth and lactation. In obese women, no additional storage is necessary, which suggests that pregnancy weight gain could be restricted because of physiological mechanisms. Our study was based on self-reported measures of weight before pregnancy whereas many studies have found that weight is more likely to be under-reported by women with a high pre-pregnancy BMI [20]. Such a bias would therefore impact on the calculation of gestational weight gain and lead to an overestimation of weight gain in overweight and obese women.

To our knowledge there is a study that examines gestational age specific weight gain as a predictor for excessive gestational weight gain. Chmitorz et al. [21 identified second trimester specific cutoffs as good predictors of excessive GWG in obese and overweight women (93.3\% PPV [95\% CI 90-96 \%]). Using second trimester cutoffs they were able to predict total excessive gestational weight gain in more than $70 \%$ of the population [21].

Gestational weight gain was significantly less in older and more parous women, a finding consistent with the existing literature [22]. In this sample, gestational weight gain decreased with the increasing age of the mother ( $25 \%$ of women between 20 and 24.9 years vs $12.5 \%$ in women of more than 35 years old, $\mathrm{p}=0.07$ ). Also, $62.5 \%$ of nulliparous, $12.5 \%$ of primiparous and $25 \%$ of multiparous women had excessive weight gain in the 2 nd trimester. Weight gain decreases with the increasing number of children $(62.5 \%$ in nulliparous women vs $25 \%$ in multiparous, $\mathrm{p}=0.02$ ).

There is evidence that physical activity along with dietary modification and weight monitoring can decrease gestational weight gain [23], [24]. A meta-analysis by Streuling et al. [23] examined 12 randomized trials assessing the impact of a physical activity intervention on GWG. Studies included had participants exercise at least three times a week for 20-60 min starting in the first or second trimester. Physical activity included aerobics, running, cycling, water aerobics and muscle strengthening. Women who engaged in any physical activity during pregnancy had a significantly lower gestational weight gain compared to those who did not (mean difference $-0.61 \mathrm{~kg} ; 95 \%$ CI -1.17, -0.06). Although exercise overall reduced total GWG the meta-analysis did not find a dose-dependent effect of exercise on gestational weight gain.

Studies have shown that both zero weight gain and weight loss are associated with decreased rates of preeclampsia, cesarean delivery, large for gestational age infants, operative vaginal deliveries, and admission to neonatal intensive care units [25],[26].

A limitation of our study is the use of self-reported weights of participants. This is important because self-reported weights may cause misclassification of gestational weight gain. Obese women tend to 
underreport weight and therefore overestimate gestational weight gain [27]. Measured pre-pregnancy weight may not be universally available and first trimester weight is often considered acceptable. Our methodology of calculating total gestational weight gain using either a self-reported weight or documented first trimester weight reflects how clinicians examine gestational weight gain in a real world setting.

\section{CONCLUSION}

In our cohort we found that women at risk for exceeding gestational weight gain goals are normal and underweight women before pregnancy rather than overweight and obese ones, less aged ( $<35$ years old), nulliparous and primiparous women comparing with multiparous ones. This finding should encourage health professionals to better inform pregnant women about the importance of weight gain adapted to their BMI.

However, as less than a third of women currently meet the recommended gestational weight gain limits by the Institute of Medicine, it is important, in a health public point of view, to set up programs to help pregnant women to comply with these recommendations.

Future research should focus on interventions that provide education regarding food choices and encourage women to engage in physical activity early in pregnancy. These interventions should specifically target women who gain more weight at the beginning of the second trimester.

\section{REFERENCES}

[1] K. M. Flegal, et al., "Prevalence of obesity and trends in the distribution of body mass index among US adults, 1999-2010,” JAMA, vol. 307, pp. 491-497, 2012.

[2] CDC, "Pregnancy nutrition surveillance nation. Summary of trends in maternal health indictors," 2011. http://www.cdc.gov/pednss/pnss_tables/tables_numeric.htm. Retrieved February 25, 2014.

[3] American College of Obstetricians and Gynecologists, "Obesity in pregnancy," ACOG Committee Opinion 549. Washington, DC, ACOG, 2013.

[4] Institute of Medicine, "Weight gain during pregnancy: Reexaming the guidelines," Washington, DC, National Academies Press, 2009.

[5] Royal College of Obstetricians and Gynaecologists, "CMACE/RCOG joint guideline: management of women with obesity in pregnancy," London, Royal College of Obstetricians and Gynecologists, 2010.

[6] American College of Obstetricians and Gynecologists, "Obesity in pregnancy. Committee Opinion No. 549. American College of Obstetricians and Gynecologists," Obstet Gynecol, vol. 121, pp. 213-217, 2013.

[7] Davies G. A., et al., "SOGC clinical practice guidelines: obesity in pregnancy. No. 239, February 2010," Int J Gynaecol Obstet, vol/issue: 110(2), pp. 167-173, 2010.

[8] D. Durie, et al., "Effect of second trimester and third-trimester rate of gestational weight gain on maternal and neonatal outcomes," Obstetrics and Gynecology, vol/issue: 118(3), pp. 569-575, 2011.

[9] Hedderson M. M., et al., "Gestational weight gain and risk of gestational diabetes mellitus," Obstet Gynecol, vol/issue: 115(3), pp. 597-604, 2010. [PubMed: 20177292].

[10] Buchanan T. A. \& Xiang A. H., "Gestational diabetes mellitus," J Clin Invest, vol/issue: 115(3), pp. 485-491, 2005. [PubMed: 15765129].

[11] "Body fat mass and the proportion of very large adipocytes in pregnant women are associated with gestational insulin resistance," Int J Obes (Lond), 2015. doi: 10.1038/ijo.2015.232.

[12] Rasmussen K. M., et al., "New guidelines for weight gain during pregnancy: what obstetrician/gynecologists should know," Curr Opin Obstet Gynecol, vol/issue: 21(6), pp. 521-526, 2009. [PubMed: 19809317].

[13] Herring S. J., et al., "Weight gain in pregnancy and risk of maternal hyperglycemia," Am J Obstet Gynecol, vol/issue: 201(1), pp. 61, el-e7, 2009. [PubMed: 19371858].

[14] Brown J. E., et al., "Variation in newborn size according to pregnancy weight change by trimester," Am J Clin Nutr, vol/issue: 76(1), pp. 205-209, 2002. [PubMed: 12081836].

[15] Edwards L. E., et al., "Pregnancy complications and birth outcomes in obese and normal-weight women: effects of gestational weight change," Obstet Gynecol, vol/issue: 87(3), pp. 389-394, 1996. [PubMed: 8598961].

[16] Claesson I. M., et al., "Consumer satisfaction with a weight-gain intervention programme for obese pregnant women," Midwifery, 2007. (Epub ahead of print).

[17] Rasmussen K. M., et al., "Recommendations for weight gain during pregnancy in the context of the obesity epidemic," Obstet Gynecol, vol/issue: 116(5), pp. 1191-1195, 2010. [PubMed: 20966705].

[18] J. A. Hutcheon, et al., "Pregnancy weight gain charts for obese and overweight women," Obesity, vol/issue: 23(3), pp. 532-535, 2015.

[19] E. A. Nohr, et al., "Pregnancy outcomes related to gestational weight gain in women defined by their body mass index, parity, height, and smoking status," American Journal of Clinical Nutrition, vol. 90, pp. 1288-1294, 2009.

[20] Rowland M. L., "Self-reported weight and height," Am J Clin Nutr, vol. 52, pp. 1125 - 1133, 1990.

[21] A. Chmitorz, et al., "Do trimester-specific cutoffs predict whether women ultimately stay within the Institute of Medicine/National Research Council guidelines for gestational weight gain? Findings of a retrospective cohort study," American Journal of Clinical Nutrition, vol/issue: 95(6), pp. 1432-1437, 2012. 
[22] Chasan T. L., et al., "Predictors of excessive and inadequate gestational weight gain in Hispanic women," Obesity (Silver Spring), vol. 16, pp. 1657-66, 2008.

[23] I. Streuling, et al., "Physical activity and gestational weight gain: A meta-analysis of intervention trials," BJOG, vol. 118, pp. 278-284, 2011.

[24] I. Streuling, et al., "Can gestational weight gain be modified by increasing physical activity and diet counseling? A meta-analysis of intervention trials," American Journal of Clinical Nutrition, vol/issue: 92(4), pp. 678-687, 2010.

[25] M. Blomber, "Maternal and neonatal outcomes among obese women with weight gain below the new Institute of Medicine recommendations," Obstetrics and Gynecology, vol. 117, pp. 1065-1070, 2011.

[26] D. W. Kiel, et al., "Gestational weight gain and pregnancy outcomes in obese women: How much is enough?" Obstetrics and Gynecology, vol. 110, pp. 752-758, 2007.

[27] A. Mandujano, et al., "Women's reported weight: Is there a discrepancy?" The Journal ofMaternalFetal\&Neonatal Medicine, vol/issue: 25(8), pp. 1395-1398, 2012. 